\title{
A systematic review of the potential role of fruit and vegetables in depression
}

\author{
C. Rooney ${ }^{1}$, M.C. McKinley ${ }^{1}$ and J.V. Woodside ${ }^{1}$ \\ ${ }^{1}$ Centre for Public Health, Queen's University Belfast, Belfast, UK, BT12 6BJ
}

Depression is a major public health problem worldwide ${ }^{(1)}$. Some studies have indicated that fruit and vegetables (FV) could potentially influence depressed mood ${ }^{(2,3)}$. This may be because FV are rich in some nutrients (e.g. antioxidants) which have previously been linked with depression ${ }^{(2)}$. The aim of this work was to conduct a systematic review of the evidence surrounding FV intake and depression.

Electronic databases (MEDLINE, EMBASE, PsycINFO and CINAHL) were searched for relevant English language articles, published from inception to May 2013. The inclusion criteria were observational and experimental studies that examined FV intake in relation to depressed mood among healthy adults. Data were extracted from relevant papers and recorded in structured tables. Papers were categorised firstly according to study design (observational/experimental), and then sub-categorised depending on whether they examined the association/effect of FV independently with depression, or the association/effect of FV, in addition to other lifestyle/dietary components, with depression.

Fifty-six studies were included (see table). Most $(\mathrm{n}=52)$ of these were observational studies. Among the observational studies that examined FV independently with depressed mood $(n=29)$, just over half $(n=15)$ reported heterogeneous findings (i.e. significant associations were detected for certain subsets of participants e.g. males/females, types of FV or particular subscales of questionnaires, but not for others). However, amongst the observational studies which examined the association between FV, alongside other lifestyle/ dietary components $(n=23)$, and depression, most $(n=16)$ reported significant associations. None of the experimental studies $(n=4)$ reported any significant effects of increased FV consumption on depressed mood.

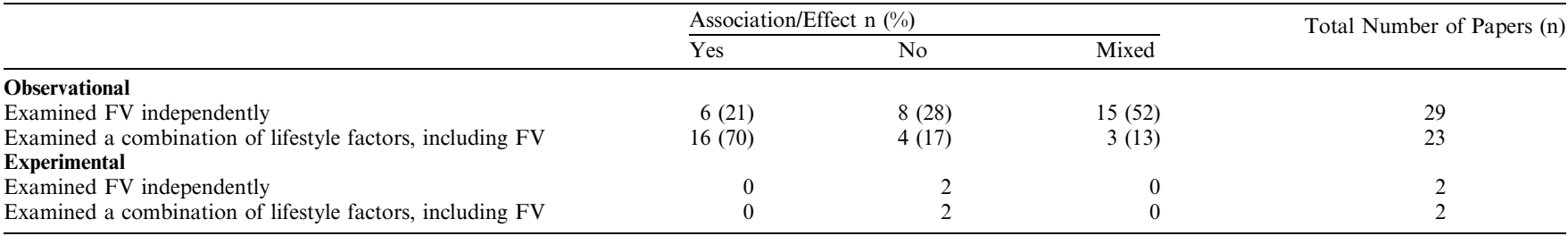

Some promising findings exist with regards to FV intake in combination with other lifestyle/dietary components and depression, but overall, results are inconsistent. Future well-designed randomised controlled trials are needed to investigate the relationship further.

1. World Health Organization. (2016) Depression. Available at: http://www.who.int/mediacentre/factsheets/fs369/en/.

2. Payne ME, Steck SE, George RR et al. (2012) J Acad Nutr Diet 112, 2022-2027.

3. McMartin SE, Jacka FN \& Colman I (2013) Prev Med 56, 225-230. 